\title{
Cost-effective solutions and tools for medical image processing and design of personalised cranioplasty implants \\ B.M. Pham ${ }^{1}$, C.H. Le ${ }^{2}$, N.T., Do ${ }^{1}$, D.L. Vo ${ }^{3}$, T. Le ${ }^{4}$, R. Lee ${ }^{5}$, M.S. Packianather ${ }^{6}$, S.Mengistu ${ }^{2}$ \\ ${ }^{1}$ Vietnamese Academy of Science and Technology, Hanoi, Vietnam \\ ${ }^{2}$ Faculty of Engineering and Science, University of Greenwich, Kent, United Kingdom \\ ${ }^{3}$ Ho Chi Minh City Medicine and Pharmacy University, Ho Chi Minh, Vietnam \\ ${ }^{4}$ International University-Vietnam National University, Ho Chi Minh, Vietnam \\ ${ }^{5}$ School of Applied Sciences, London South Bank University, London, United Kingdom \\ ${ }^{6}$ School of Engineering, Cardiff University, Cardiff, United Kingdom
}

\begin{abstract}
Cranial defects which are caused by bone tumors or traffic accidents are treated by cranioplasty techniques. Cranioplasty implants are required to protect the underlying brain, correct major aesthetic deformities, or both. With the rapid development of computer graphics, medical image processing (MIP) and manufacturing technologies in recent decades, nowadays, personalised cranioplasty implants can be designed and made to improve the quality of cranial defect treatments. However, software tools for MIP and 3D modelling of implants are expensive; and they normally require high technical skills. Especially, the process of design and development of personalised cranioplasty implants normally requires a multidisciplinary team, including experts in MIP, 3D design and modelling, and Biomedical Engineering; this leads to challenges and difficulties for technology transfers and implementations in hospitals. This research is aimed at developing, in particular, cost-effective solutions and tools for design and modeling of personalised cranioplasty implants, and to simplify the design and modelling of implants, as well as to reduce the design and modeling time. In this way, surgeons and engineers can conveniently and easily design personalised cranioplasty implants, without the need of using complex MIP and CAD tools; and as a result the cost of implants will be minimised.
\end{abstract}

Keywords - Computerised Tomography (CT), Magnetic Resonance Imaging (MRI), Medical Image Processing, cranial defects, cranioplasty implants.

\section{INTRODUCTION}

Cranial defects which are caused by bone tumors or traffic accidents are treated by cranioplasty techniques. Cranioplasty implants are required to protect the underlying brain, correct major aesthetic deformities, or both. With the rapid development of computer graphics, Medical Image Processing (MIP) and manufacturing technologies in recent decades, especially the advancements of (Computer Aided Design/Computer Aided Manufacturing/Computer Numerical Control (CAD/CAM/CNC), Reverse Engineering (RE), and Rapid Prototyping and Manufacturing (RP\&M), nowadays, personalised cranioplasty implants can be designed and made to improve the quality of cranial defect treatments [1-5]. Although benefits of using personalised cranioplasty implants are well recognised, the number of clinical cases at hospitals is still limited, and this state-of-the-art treatment technology has not been widely applied due to difficulties of technology transfer to hospitals, high requirements of technical skills, and a high cost of personalised implants. The main reasons that lead to these difficulties include [1]: (1) Complexity of the implant design, (2) Challenges about multi-disciplinary collaboration \& communication, and (3) High cost of technology. For the countries with low income, the cost-effective solutions for diagnosis and treatments are very important, and the cost of implants is one of the key criteria in the decision making process. The cost range of the personalised cranioplasty implants with the size of $150 \times 165 \mathrm{~mm}$ is approximately from 300 USD to 4000 USD [5], depending on the biomaterials of implants (Bone cements, PMMA, Titanium alloys) and the complexity of cranial defects. In addition, advanced technologies for design and development of medical personalised products are expensive and not always available in the developing countries.

This research is aimed at developing, in particular, costeffective solutions and tools for design and modeling of personalised cranioplasty implants, with the focus on the following objectives which address the above presented challenges: (1) Ease of use and high effectiveness, and (2) Cost-effective solutions. The design software tools and technological solutions should be convenient and simple to use. There is basically no need to use complex MIP and CAD as well as RE tools. Medical doctors or engineers should both be able to work on the implant design independently or with a fewer requirements about close collaborations and communications during the implant design and product development (DPD) process. The MIP and 3D CAD modeling software tools could be in-house developed for the specific types of applications. In this way, the cost of implants will be minimised.

\section{Methods}

The workflow and methods for design and modelling of personalised cranioplasty are presented in Fig.1. The input data for development of cranioplasty implants is CT/MRI 
images in the form of DICOM data format. CT images are commonly used for bone reconstructions. First of all, a defective skull is reconstructed. Then, a boundary of a cranial defect is determined. The boundary of a cranial defect and an intact area of a skull are used as the input and guide to create an implant template which is an approximate shape (draft design) of an implant. Finally, the implant template is controlled and modified in details to obtain the optimal shape of an implant. The following are the details of the algorithms and techniques used for modelling cranioplasty implants as shown in Fig.1.

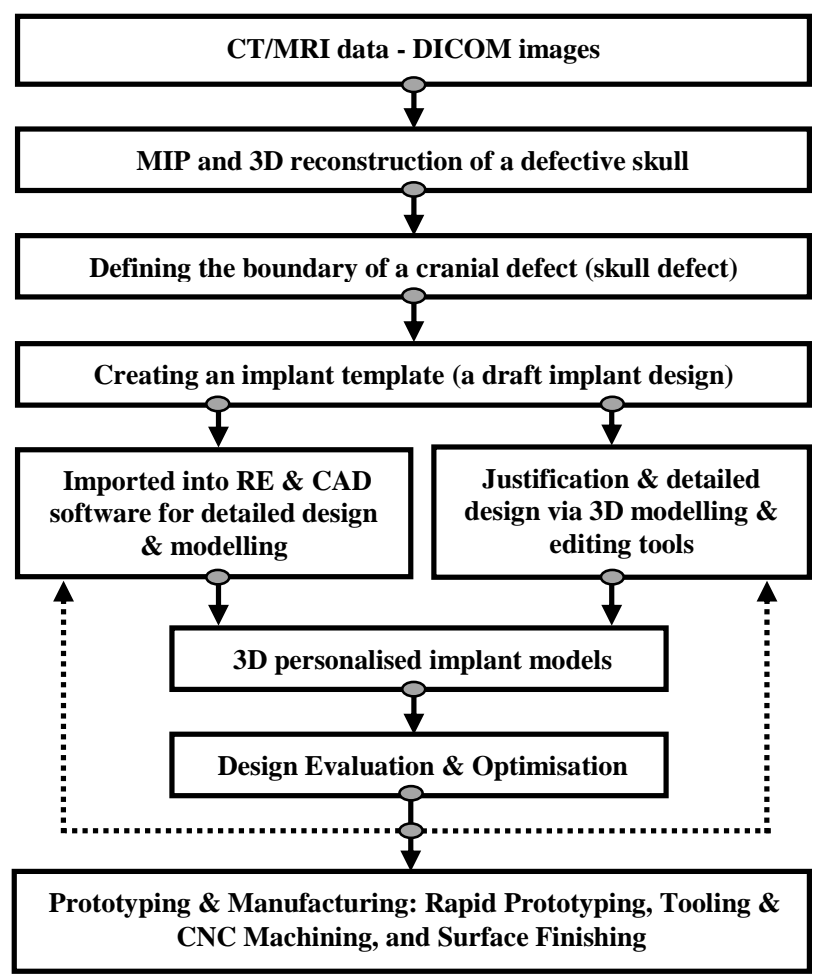

Fig.1 Methods for development of personalised cranioplasty implants

\section{A. STEP 1: Medical Image Processing $-3 D$ reconstructions of a defective skull}

Marching Cube (MC) is an efficient method for extracting isosurfaces from 3D scalar field [6]. The MC algorithm is used in this study to reconstruct 3D models of a defective skull. The following are the key points of the applied marching cubes algorithm. (a) Inputs: CT images in the form of DICOM format. Each CT slice image has $(\mathrm{MxN})$ pixels, and the gray level of images. (b) Outputs: 3D models of bone structure (skulls). (c) Algorithm: A DICOM format does specify a lot of Transfer Syntaxes. For the compressed Transfer Syntaxes, the Meta data information is encoded using Explicit VR and Little Endian. The biggest challenge in the DICOM image processing is how to analyze and segment the data Area of Interest (AoI) such as bone, soft tissue, water, etc. In order to do this, the data conversion is required, based on the following two steps. (1) Step 1: The pixel data values are converted into Hounsfield values $(H U)$ : For CT Images, the relationship between the Stored Values (SV) and the Hounsfield values is defined by the following formula: $H U=S V *$ RescaleSlope $+\mathrm{Re}$ scaleIntercept. RescaleSlope and RescaleIntercept are retrieved from the meta data section of the DICOM file. (2) Step 2: The Hounsfield values are converted into the grayscale values: The stored values (SV) are converted into Hounsfield values (HU), the Hounsfield values are then converted further into meaningful grayscale values (Fig. 2(a)). The Hounsfield values range from -500 (e.g., lung) to +800 (e.g., bone).

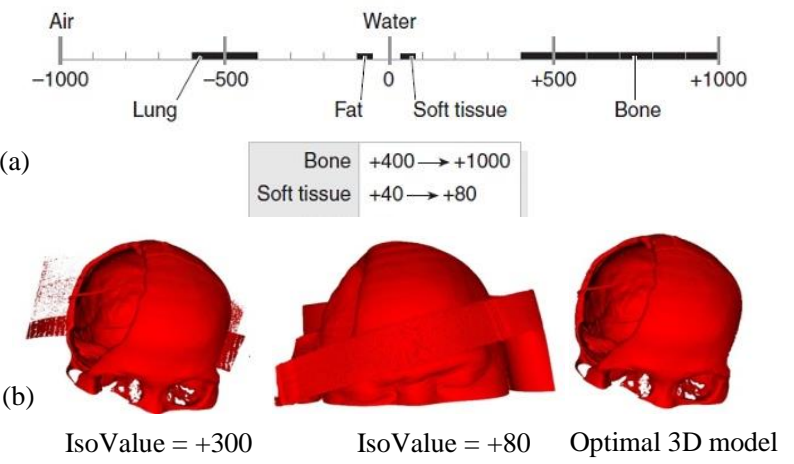

Fig. 2 (a): Grayscale for image segmentations. (b): 3D models of a skull and head reconstructed from DICOM images with different gray scales (IsoValue).

After conducting the image analysis and segmentation based on the grayscale values, 3D models of AoI are reconstructed from the serial CT images, based on the Marching Cubes algorithm [6, 7]. The algorithm is implemented as follows. (a) Inputs: Serial CT images (x images - each image has mn pixels), threshold grayscale value (IsoValue). (b) Outputs: 3D models of AoI (M). (c) Algorithm: The main content of the algorithm is as follows:

1. $M=$ Null.

2. For each Voxel of the CT Slice

2.1. An own GridCell (GC) is created, GC is a cube.

2.2. If all the vertex value of $G C>I s o V a l u e$ or $<$ IsoValue, then GC is not selected.

2.3. If there exist the vertex value of $G C>$ IsoValue and also exist the value < IsoVale, then all the vertex value of $G C$ is selected. $M=M \cap T$, where $T$ is the vertices value of $G C$.

3. Optimize $M$.

C\# was used in combination with the ISG graphics library which was developed in-house based on the OpenGL graphics library. A series of tests were done to verify the algorithm and accuracies of the 3D reconstructed models. 
Figure 2 (b) presents a typical example of 3D models of a head and skulls reconstructed form CT data which consists of 196 images; and each image has a size of $512 \times 512$ pixels.

\section{B. STEP 2: Defining the boundary of a cranial defect and construction of an implant template}

Determining an accurate cranial defect window plays an important role in design of an optimal implant. We developed a tool for defining a boundary of a defective window. A defect boundary is manually defined by a mouse. Each click of a mouse is a computational process between the direction vector $\mathrm{V}_{i}$ which is generated by the vector direction of the camera in conjunction with the coordinates of the cursor, in collision (intersection) with the surface of a 3D skull model. The result is a point $\left(\mathrm{P}_{i}\right)$ as shown in Fig. 3(a).

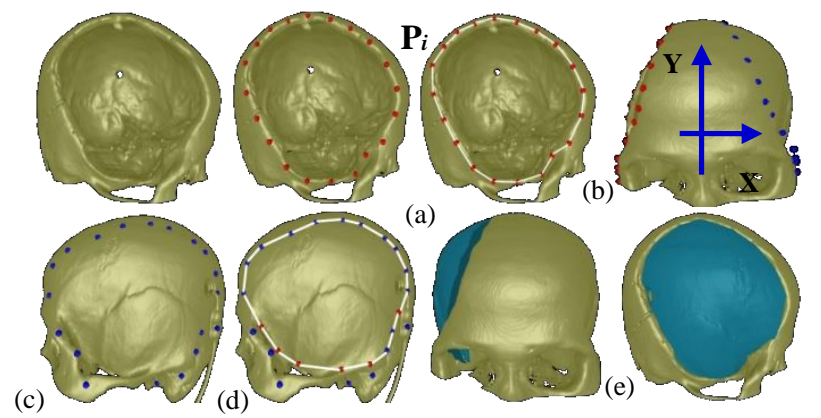

Fig. 3 (a) Definitions of a boundary of a defective window. (b, c) Identification of a symmetrical intact area. (d) Optimal symmetrical intact area.

(e): An implant template created based on a symmetrical intact area.

Related methods for modeling cranioplasty implants are well documented in [3-5]. The construction of an implant template (the $1^{\text {st }}$ version or a draft implant model) for the MIP and 3D modeling tools in this study is based on mirrored surfaces of a symmetrical intact area of a defective skull. For each point on the defective boundary (Fig.3(a), RED dots), a "symmetrical" point in the intact area (Fig.3(b), BLUE dots) could be found. Since a skull geometry normally is not $100 \%$ symmetrical; the created symmetrical points may not lie on a skull surface. This problem could be solved via the 2 following steps. (1) Step 1: Create a Blue point $\left(\mathrm{P}^{\prime} \mathrm{i}\right)$ which is a symmetrical point of a RED point $(\mathrm{Pi})$ through a symmetrical axis Y. $\mathrm{P}^{\prime} \mathrm{i}$ is calculated via $\mathrm{Pi}^{\prime} . \mathrm{x}=-\mathrm{Pi} . \mathrm{x}$. The point P'i is not always on a skull surface. (2) Step 2: Recalculate $\mathrm{P}_{i}^{\prime}$ by using a vector $\mathrm{V}_{i}^{\prime}$ which is a symmetrical vector of a vector $\mathrm{V}_{i}$. The intersection between $\mathrm{V}^{\prime}{ }_{i}$ and the intact skull surface is the point P'i that is of interest. Finally, the symmetrical area of a cranial defect could be defined as shown in Fig.3(c) in which the red points are the recalculated ones. Hence, an implant template could be obtained as shown in Fig.3 (d). This implant template can be conveniently modified and edited in RE or
CAD software to obtain the optimal one with a better accuracy and design features. In case, there is no RE or CAD tools available, an optimal design could be obtained using an additional 3D editing tool described in STEP 3.

\section{STEP 3: Justification \& detailed design via 3D model- ling tools}

In this step, an implant template is further adjusted and controlled to obtain the required form and shape of an implant. The adjustment is done by manipulating the surfaces for an optimal shape and a better contact between an implant and a skull via the following 2 steps: (1) Definitions of control points shown in Fig. 4(a, b), and (2) Detailed adjusts, edit and controls of an implant model. Control points of an implant are created by a separate editing tool based on CT images as shown in Fig. 4(c-f). (a)
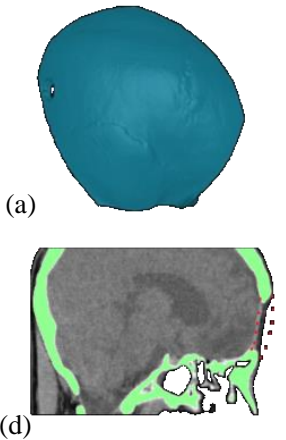

(b)

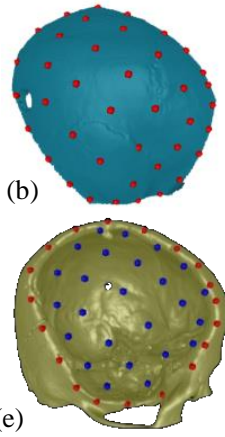

(c)

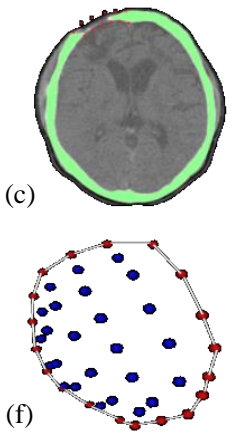

Fig. 4 (a,b) Control points of an implant (c,d) Defining control points on CT images. Inputs for the RBFs algorithm: Outer and inner surfaces of an implant template (a), Control points of an implant template (b), and Control points of a target implant $(e, f)$.

A 3D modeling and editing tool is developed for adjusting and controlling an implant template to obtain an optimal form and shape based on the Radial Basis Function (RBF) [8] which is used for creating a smooth interpolation between the values known only at a discrete set of positions. The inputs for the RBFs algorithm are shown in Fig. 4(b, f). It is assumed that the deformation of an implant when adjusted and controlled is known for $n$ 3D positions $x_{i}$ and this information is represented by a vector describing a 3D displacement $u_{i}$ of the geometry that is positioned at $x_{i}$ in the original and undeformed state. The $x_{i}$ positions are considered as the control points that have been moved to positions $x_{i}+u_{i}$. Specifically, there are two steps of adjusting and controlling the implant template to be the optimal shape (target implant). The first step is to determine the transform function $\mathrm{F}$, is a mapping from the control points shown in Fig.4(b) to the control points shown in Fig.4(e, f). The second step is to apply the function $\mathrm{F}$ with all the data on the implant template model shown in Fig.4(a). The RBF interpolation method is now used to transform these points to the new positions in order to obtain a target implant with the optimal shape as expected. 


\section{STEP 4: Design evaluation \& optimisation}

An optimal model of an implant constructed in STEP 3 is finally checked and evaluated so that it meets well both technical and clinical requirements. Figure 5(h) presents the evaluation of an implant thickness and a contact between an implant and a skull. Surgeons (medical doctors) are normally involved in this step. Design optimisation via the use of Computer Aided Engineering (CAE) is done when necessary to assess the mechanical strength, design for manufacturing issues, as well as the use of different biomaterials for fabrication.

\section{Results AND CONCLUSIONS}

A typical result of designing personalised cranioplasty implants based on the developed MIP and 3D modeling software tools and methods mentioned in Section II is presented in Fig.5. In this case study, the defective skull does not have a good symmetry. However, the developed MIP and modeling tools are still effectively used for modelling an implant which meets well the technical and clinical requirement.

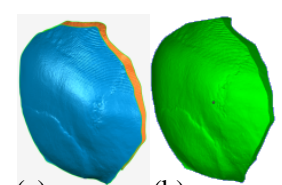

(a)

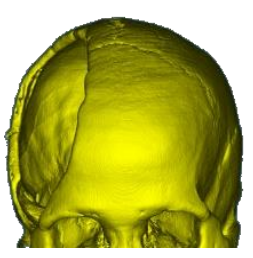

(f)

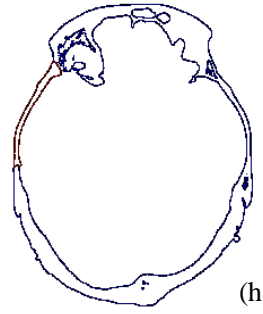

(c)

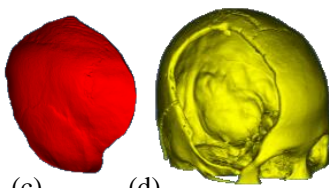

(d)
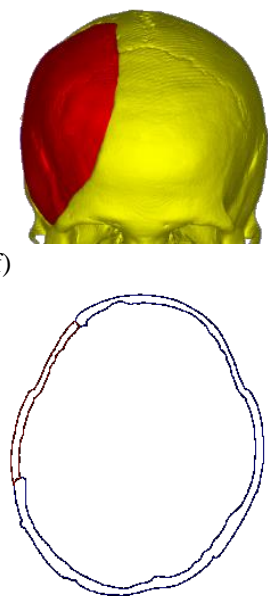

(g)

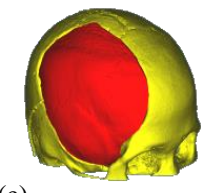

(e)
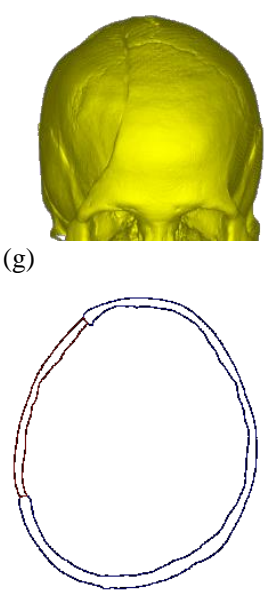

Fig. 5 (a) External and internal surface of an implant. (b): A first design of an implant. (c) A final implant model. (e ,f, g) A defective skulls and designed implant. (h): Design verification and evaluation: Checking the contact between an implant (RED) and a defective skull (BLUE).

Basically, it takes about 2-4 hours to complete design of a cranioplasty implant, from MIP to 3D design and modeling of an implant. Based on the draft model of an implant which is directly generated by the developed modeling tools
(Fig. 5(a, b)), it takes about an additional 10-15 minutes to obtain an optimal implant model with the quality that is similar to the one that is designed based on the currently available MIP and RE packages in the market (Fig. 5(c-g)).

With the in-house developed MIP and modeling software packages, there is basically no need for additional RE and CAD software packages when working on the design and development of cranioplasty implants. The use of these MIP and modeling tools is not complex; surgeons and medical doctors with no strong background in CAD and 3D modeling are able to design cranioplasty implants. In this way, the cost of implants is minimised; and the technology transfer to hospitals is made more convenient. The next versions of this MIP and 3D modelling software will focus on solving the issues and challenges related to design and modelling of personalised implants for the cases in which the reference data or the symmetrical features are not available for the design. The future work will consider the workflow for optimizing the process of designing personalised cranioplasty implants in order to reduce the costs further. These software tools are to be integrated into the national openarchitecture MIP and CAD system which is used for design and development of patient and population-specific medical products and services.

\section{ACKNOWLEDGMENT}

British Council-Newton Fund is acknowledged for their support.

\section{REFERENCES}

1. Hieu L.C. et al. (2010) Integrated approaches for personalised craniomaxillofacial implant design and manufacturing, IFMBE Proc. vol. 27, 2010, pp 119-122

2. Hieu L.C. at al. (2005) Medical rapid prototyping applications and methods. Assembly Automation; 25(4): 284-292

3. Hieu L.C. et al. (2004) A cheap technical solution for cranioplasty treatments. Technology and Health Care; 12(3): 281-292

4. Hieu L.C et al. (2003) Design for medical rapid prototyping of cranioplasty implants, Rapid Prototyping Journal; 9(3): 175-186

5. Hieu L.C. at al. (2002) Design and manufacturing of cranioplasty implant by 3 -axis CNC milling. Technology and Health Care; 10(5): 413-23

6. C. Dyken et al. (2008) High-speed marching cubes using histogram pyramids, Computer Graphics Forum; 27(8) 2028-2039.

7. William E. Lorensen and Harvey E. Cline (1987) Marching Cubes: A high resolution 3D surface construction algorithm. Computer Graphics; 21 (4): 163-169

8. Powell MJD (1992) The theory of radial basis function approximation. In: Will Light (Editor) Advances in Numerical Analysis, Vol. II: Wavelets, Subdivision Algorithms and Radial Functions, Oxford University Press, Oxford, UK, 105-210.

Author: Pham Ba May

Institute: Vietnamese Academy of Science and Technology

Street: Hoang Quoc Viet

City: Hanoi

Country: Vietnam

Email: maypb@ioit.ac.vn 